\title{
Analysis and Research on Acid Oil's Esterified Deacidification
}

\section{Based on Chemometrics}

\author{
aLi-Tao Jiao, bjian Yang \\ No.3111, Linghai Road, Huangdao District, Qingdao City, Shandong Province, China \\ aJiaolitao_11@163.com \\ byangjian8482@163.com
}

Keywords: Biodiesel, High acid value oil, Esterification, Catalyst

\begin{abstract}
This article mainly researched esterified deacidification of biodiesel raw materials waste oil, with chemometrics method. It also analyzed and researched esterified deacidification of methanol, glycerin , and their mixture under the condition of sulfuric acid, sodium hydrogen sulfate, titanium sulfate as catalyst through a large number of experiments. the corresponding dropping acid curve was mapped and compared. The results showed that sulfuric acid catalytic effect is better, then is the titanium sulfate. Through experiments, this article explored and summarized secondary esterification process of sulfuric acid, sodium hydrogen sulfate, titanium sulfate, three kinds of catalyst catalyzing methanol respectively.
\end{abstract}

\section{Introduction}

Chemometrics is the science of extracting information from chemical systems by data-driven means. Chemometrics is inherently interdisciplinary, using methods frequently employed in core data-analytic disciplines such as multivariate statistics, applied mathematics, and computer science, in order to address problems in chemistry, biochemistry, medicine, biology and chemical engineering. This article mainly research esterified deacidification of biodiesel raw materials waste oil, using the chemometrics method, analyzed and researched esterified deacidification of methanol, glycerin , and their mixture under the condition of sulfuric acid, sodium hydrogen sulfate, titanium sulfate as catalyst through a large number of experiment and Data calculation and analysis.

\section{Experiment reagents and equipment}

The reagents used in the experiment

Raw material oil: Acid oil (after dehydration pretreatment)

Anhydrous methanol (Tianjin Guang Fu Fine Chemicals Institute, AR)

Anhydrous ethanol(Tianjin Da Mao Chemical Reagent Factory, AR)

Concentrated sulfuric acid (Shandong Laiyang Feng Shan Chemical plant,95\%-98\%，AR)

Sodium hydroxide(Tianjin Kai Tong Chemical reagent co., LTD,AR)

Aether(Tianjin Bei Hong Reagent Factory, AR )

Titanium sulfate(Sinopharm Group Chemical reagent co., LTD,CP)

Monohydrate sodium bisulfate (China Elaboration Science and Technology Institute)

Phenolphthalein(Tianjin Da Mao Chemical Reagent Factory)

\section{Experiment equipment}

KDM type air-conditioning electric sets（Longkou Xian KE Instruments inc.) 
Electric blender(Jintan Jin Cheng Teaching Instrument Factory, Power40W, Max Speed 3000r/min)

Rotary evaporator (Shanghai Ya Rong Biochemical Instrument Factory)

Electronic Analytical Balance(Shanghai Min Qiao Scientific instrument co., LTD, Model SL512)

Condenser pipe, hob, thermometer, frosted three flask $(500 \mathrm{ml})$, long neck dropper, alkali type buret, pipette $(20 \mathrm{ml})$, and 2 beakers $(100 \mathrm{ml})$

\section{Experimental process}

\section{Acid value measurement method}

(1)Experimental principle

Natural oils will stink in the air for a long time, this phenomenon is called rancidity. Rancidity is due to water release free fatty acids, fatty was oxidized to aldehydes or ketones in the air, which has a certain stink smell. [1] The degree of rancidity is represented by acid value. Acid value is mg number of $\mathrm{KOH}$ which is required by free fatty acids of neutralized $1 \mathrm{~g}$ fat.

Grease neutralization reaction of free fatty acid and $\mathrm{KOH}$, from $\mathrm{KOH}$ consumption standard solution to calculate the amount of free fatty acids.

Equation is as follows:

(2)Experimental operation

$$
\mathrm{RCOOH}+\mathrm{KOH} \rightarrow \mathrm{RCOOK}+\mathrm{H}_{2} \mathrm{O}
$$

Accurately weigh $0.5 \mathrm{~g}$ fat in conical flas $(100 \mathrm{ml})$, add alcohol ether mixture $20 \mathrm{ml}$, shake to dissolve or $40{ }^{\circ} \mathrm{C}$ water bath melts to transparent.The solution turns red to colorless, continue to use $0.1 \mathrm{~mol} / \mathrm{L} \mathrm{KOH}$ standard liquid, then the droplets to reddish, after $1 \mathrm{~min}$ it is not fade for the finish line, and record the dosage amount of $\mathrm{KOH} \mathrm{V}(\mathrm{ml})$.

(3)Experimental calculation

$$
\begin{aligned}
& \text { acid value of fat }==56.1 \times \mathrm{cV} / \mathrm{m} \\
& \text { in the formula } \mathrm{c}: \text { concentration of standard } \mathrm{KOH} \text { material } \\
& \mathrm{V}: \mathrm{KOH} \text { volume of sample consumption }(\mathrm{ml}) \\
& 56.1: \text { mass of per mole } \mathrm{KOH} \\
& \mathrm{m}: \text { sample mass }(\mathrm{g})
\end{aligned}
$$

Determine acid value of high acidity oil used in the experiment with the established acid value determination system , and record as raw data of experiment:A.V.=140

\section{Research of methanol deacidification}

This experiment takes concentrated sulfuric acid (1\%), monohydrate sodium hydrogen sulfate $(4 \%)$, titanium sulfate $(3 \%)$ as catalyst, methanol and oil acid as reactant to make deacidificatiion.

Reaction system:Take $20.0 \mathrm{~g}$ acid oil of dehydration preprocessing, adding different amount of methanol (20\%), appropriate amount of catalyst, after mixing drain to three flask with a glass rod. After opening the cooling water then heating, open mixing device for esterification reaction. Every half an hour take sample to determine acid value of acid oil , until acid value is stable.

Equation is as follows:

$$
\mathrm{RCOOH}+\mathrm{CH}_{3} \mathrm{OH} \rightarrow \mathrm{RCOOCH}{ }_{2}+\mathrm{H}_{2} \mathrm{O}
$$

Noticing in the experiment:

(1)Adding order of the reagent: after mixing the oil with methanol then adding by drops of concentrated sulfuric acid

(2)Pay attention to the sealing and temperature control of the whole experiment device to prevent excessive oxidation of reaction liquid.[2] 


\section{Research of glycerin deacidification}

This experiment takes sulfuric acid $(0.5 \%, 1 \%, 1.5 \%)$, monohydrate sodium hydrogen sulfate $(4 \%)$, titanium sulfate $(3 \%)$ as catalyst, glycerin and oil acid as reactant to make deacidificatiion.

Reaction system:Take $20.0 \mathrm{~g}$ acid oil of dehydration preprocessing, adding glycerin (20\%)and appropriate amount of catalyst, after mixing drain to three flask with a glass rod. After opening the cooling water then heating, open mixing device for reflux condensation esterification reaction. Every half an hour take sample to determine acid value of acid oil, until acid value is stable.

Esterification reaction of sulfuric acid as catalyst when acid value drop is stable, add sodium hydroxide corresponding to the amount of sulfuric acid to neutralize, then add $30 \%$ amount of methanol of $1 \%$ sodium hydroxide solution, heating and stirring to react for an hour, fluid, to determine the sublayer quality.

For monohydrate sodium hydrogen sulfate, titanium sulfate catalytic reaction, when acid value drop is stable, add $20 \%$ methanol to heat and stir to make reflux condensation reaction. Determine acid value at the interval of half an hour, until acid value is stable.[3]

Noticing in the experiment:

(1)Evaporate off water generated in the reaction to make positive esterification reaction, glycerin drop acid reaction is direct open-cup.

(2)Fully stirred in the reaction process to ensure sufficient mass transfer.

\section{Single deacidification research of methanol and glycerin mixture}

This experiment takes sulfuric acid $(1.5 \%)$, monohydrate sodium hydrogen sulfate (4\%), titanium sulfate $(3 \%)$ as catalyst, methanol, glycerin and oil acid as reactant to make deacidificatiion.

Reaction system:Take $20.0 \mathrm{~g}$ acid oil of dehydration preprocessing, adding methanol (20\%),glycerin with appropriate ratio(methanol and glycerin quality ratio is: $1 / 0.547$ ) and appropriate amount of catalyst, after mixing drain to three flask with a glass rod. After opening the cooling water then heating, open mixing device for reflux condensation esterification reaction. Every half an hour take sample to determine acid value of acid oil , until acid value is stable.

\section{Secondary esterification process optimization research of methanol and glycerin mixture}

On the basis of single deacidification research of methanol and glycerin mixture, this experiment selects a specific acid value (time) as a reference point and adopts secondary esterification to drop acid.[4]

Reaction system:Take $20.0 \mathrm{~g}$ acid oil of dehydration preprocessing, adding recycling methanol glycerin $(20 \%)$,after mixing drain to three flask with a glass rod. After opening the cooling water then heating, open mixing device for reflux condensation esterification reaction. Every half an hour take sample to determine acid value of acid oil, until acid value is stable.

After single deacidification reaction, do liquid points and take upper oil, add methanol $(20 \%)$, continue to esterification reaction, tracking sample to determine acid value. According to acid value changes of the two esterification to make further optimization process and adjust datum. In the second esterification, adopt to add methanol glycerin and methanol reaction directly, and add glycerin settlement two ways to compare.

\section{The analysis and discussion of experiment results Research of methanol deacidification}

This experiment takes concentrated sulfuric acid (1\%), monohydrate sodium hydrogen sulfate $(4 \%)$, titanium sulfate $(3 \%)$ as catalyst, methanol and oil acid as reactant to make deacidification, See Table 1 and Fig.1. 


\section{The experimental data of $\mathbf{2 0 \%}$ amount of methanol deacidification as follows}

Table 1 methanol esterification research data table(20\% methanol )

Notice: time:hour; acid value unit is $\mathrm{mg} / \mathrm{g}$,according to $\mathrm{KOH}$; atalyst sodium bisulfate is monohydrate sodium bisulfate.

\begin{tabular}{|c|c|c|c|c|c|c|c|c|c|c|c|c|}
\hline NO. & Catalyst & 0 & 0.5 & 1 & 1.5 & 2 & 2.5 & 3 & 3.5 & 4 & 4.5 & 5 \\
\hline 1 & Sulfuric acid & 133 & 43 & 33 & 27 & 28 & 23 & 24 & 20 & 21 & 21 & 16 \\
\hline 2 & sodium bisulfate & 135 & 91 & 73 & 65 & 56 & 60 & 51 & 44 & 46 & 47 & \\
\hline 3 & titanium sulfate & 135 & 63 & 44 & 35 & 41 & 30 & 26 & 22 & 18 & 23 & \\
\hline
\end{tabular}

Deacidification of $1.5 \%$ sulfuric acidcatalyzing $20 \%$ methanolDeacidification of $4 \%$ monohydrate sodiumbisulfatecatalyzing $20 \%$ methanol

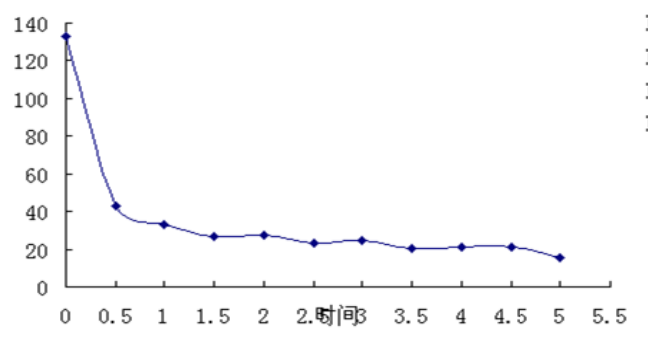

Chart 1

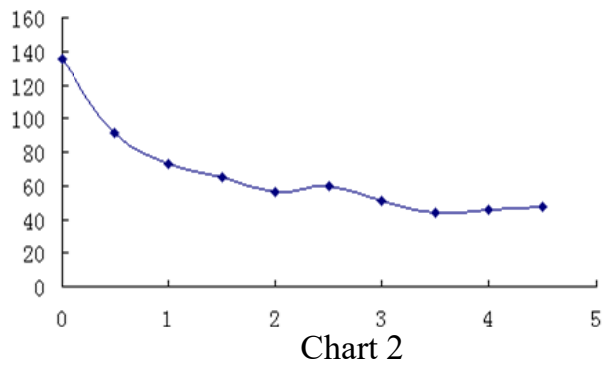

Deacidification of $3 \%$ titanium sulfate catalyzing $20 \%$ methanol

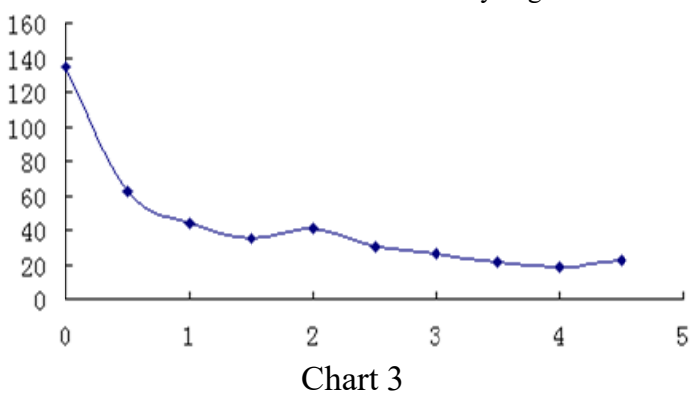

Fig. 1 Trend line changes with time of $20 \%$ amount of methanol deacidification acid value (x axis is time, $y$ axis is acid value)

\section{The discussion of experimental results analysis}

Through the above three groups of experimental data and acid value change trend line analysis, we find that:

a.The catalytic deacidification reaction speed of sulfate acid is fast, the best effect, then titanium sulfate, followed by a hydrated sodium bisulfate.[5]

b.Acid value decrease speed of three kinds of catalyst within the first hour reaction is fast (sulfate, titanium sulfate is most obvious), acid value decrease more slowly after an hour.

c.Sulfuric acid catalyzes 20\% methanol and high acid oil (acid value around 140) esterification can drop to 16 , monohydrate sodium bisulfate catalyzes $20 \%$ methanol with high acid oil( acid value around 140) esterification drop to 44, titanium sulfate catalyzes $20 \%$ methanol and high acid oil (acid value around 140) esterification drop to 22.(See Table 2 and Fig.2)

\section{Research of glycerin deacidification}

This experiment takes sulfuric acid (1\%), monohydrate sodium hydrogen sulfate $(4 \%)$, titanium sulfate $(3 \%)$ as catalyst, glycerin and oil acid as reactant to make deacidificatiion.

The experimental data of $20 \%$ amount of glycerin deacidification as follows

Table 2 glycerin esterification research data table $(20 \%$ glycerin $)$

\begin{tabular}{|c|c|c|c|c|c|c|c|c|c|c|c|}
\hline $\begin{array}{c}\text { No } \\
\cdot\end{array}$ & Catalyst & 0 & 0.5 & 1 & 1.5 & 2 & 2.5 & 3 & 3.5 & 4 & 4.5 \\
\hline 7 & $1 \%$ Sulfuric acid & 145 & 17 & 9 & 11 & 9 & & & & & \\
\hline 8 & Sodium bisulfate & 129 & 115 & 112 & 102 & 102 & 104 & & & & \\
\hline 9 & Titanium sulfate & 129 & 126 & 117 & 107 & 99 & 100 & 93 & 92 & 89 & 87 \\
\hline
\end{tabular}


Notice: time:hour; acid value unit is $\mathrm{mg} / \mathrm{g}$,according to $\mathrm{KOH} ; 4 \%$ sodium bisulfate catalyzes after reaction stability and add 30\% methanol; $3 \%$ titanium sulfate catalyzes reaction stability and add 30\% methanol; Catalyst sodium bisulfate is monohydrate sodium bisulfate.

$1.5 \%$ sulfuric acid catalyzes to biodiesel, add liquid $16.22 \mathrm{~g}$, get lower liquid $15.40 \mathrm{~g}$; $1 \%$ sulfuric acid catalyzes to biodiesel, add liquid15.92 g, get lower liquid $15.01 \mathrm{~g}$, so experience the amount of Separatory liquidfluid is about $94 \%$ of the adding.

Deacidification of $1 \%$ sulfuric acidcatalyzing $20 \%$ glycerinDeacidification of $4 \%$ monohydrate sodiumbisulfatecatalyzing $20 \%$ glycerin
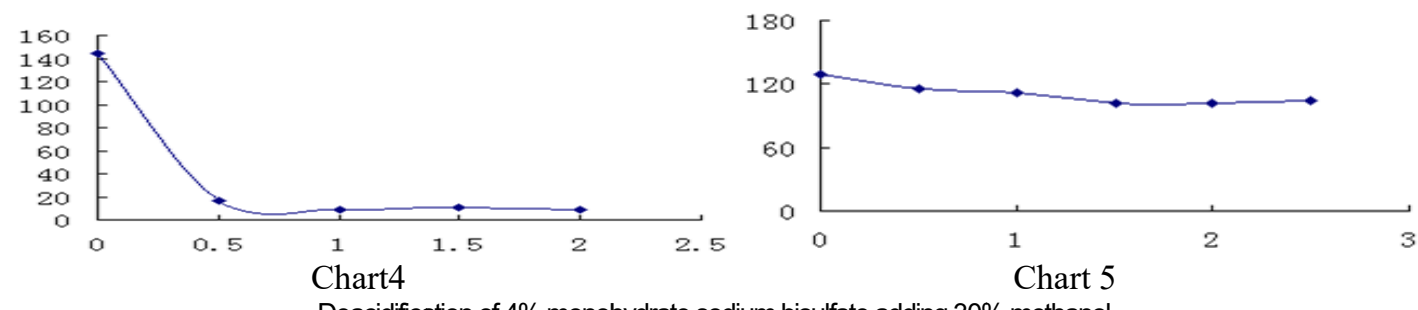

Deacidification of $4 \%$ monohydrate sodium bisulfate adding $30 \%$ methanol

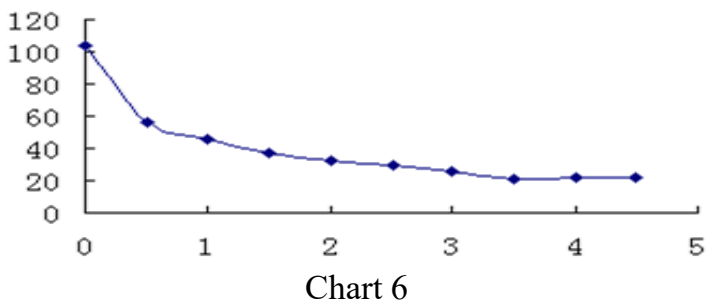

Fig. 2 Trend line changes with time of $20 \%$ amount of glycerine deacidification acid value (x axis is time, y axis is acid value)

The discussion of experimental results analysis

On the basis of the experimental phenomena and data, we analyze to get:

(1) There are side reaction in sulfate, titanium sulfate, monohydrate sodium bisulfate catalyzed glycerine esterification alone, which make 2 reaction liquid turn black and separatory liquid inconvenient. Sulfuric acid catalytic turn black fastest, then titanium sulfate, monohydrate sodium bisulfate slowest. The slower turning back the less amount of sulfuric acid.[6]

(2) Sulfuric acid catalytic deacidification speed is the fastest. Under the suitable reaction conditions, the dosage of $0.5 \%, 1 \%, 1.5 \%$ sulfuric acid as catalyst can make acid value drop from about 130 to 10 within two hours.

(3) After Glycerin drops acid in sulfuric acid catalytic conditions, add sodium hydroxide to catalyze methanol in the ester exchange reaction can produce biodiesel, we can seperate lower to obtain biodiesel according to the empirical data.

\section{Single deacidification research of methanol and glycerin mixture}

This experiment takes sulfuric acid $(1.5 \%)$, monohydrate sodium hydrogen sulfate (4\%), titanium sulfate $(3 \%)$ as catalyst, methanol $(20 \%, 30 \%)$ and appropriate amount of glycerin (methanol: glycerin $=1 \mathrm{~g}: 0.547 \mathrm{~g}$ ) and acid oil as a reactant for reaction.(See Table 3 and Fig.3)

The experimental data of $20 \%$ amount of methanol andappropriate amount of glycerin deacidification

Table 3 Single deacidification research data table of methanol and glycerin mixture (20\% methanol )

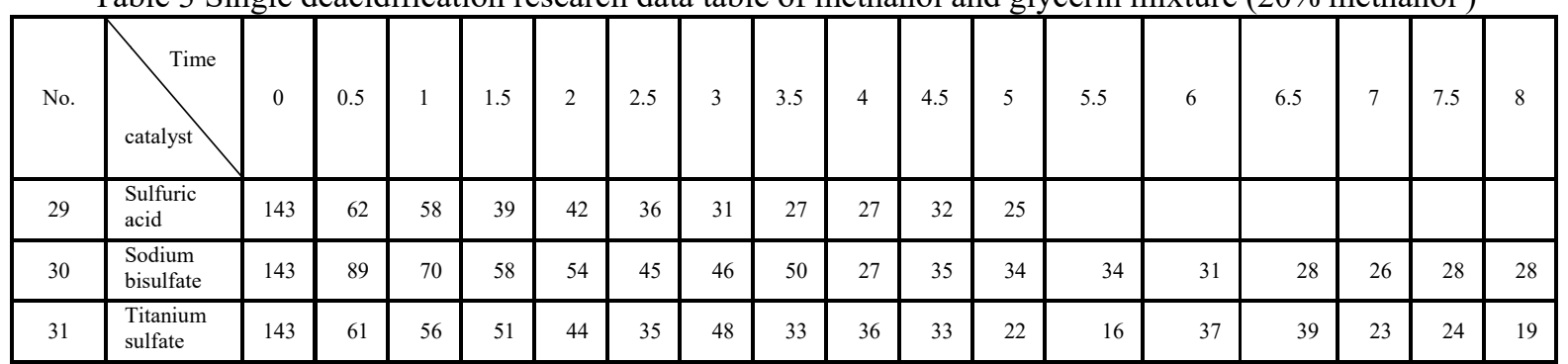


Notice: time:hour; acid value unit is $\mathrm{mg} / \mathrm{g}$, according to $\mathrm{KOH}$; Acid value of sodium bisulfate monohydrate catalyzing is $32 \mathrm{mg} / \mathrm{g}$ at 8.5 hours, titanium sulfate is $29 \mathrm{mg} / \mathrm{g}$; Catalyst sodium bisulfate is monohydrate sodium bisulfate.

Deacidification of $1.5 \%$ sulfuric acidcatalyzing $20 \%$ glycerinDeacidification of $4 \%$ monohydrate sodiumbisulfatecatalyzing $20 \%$ methanol
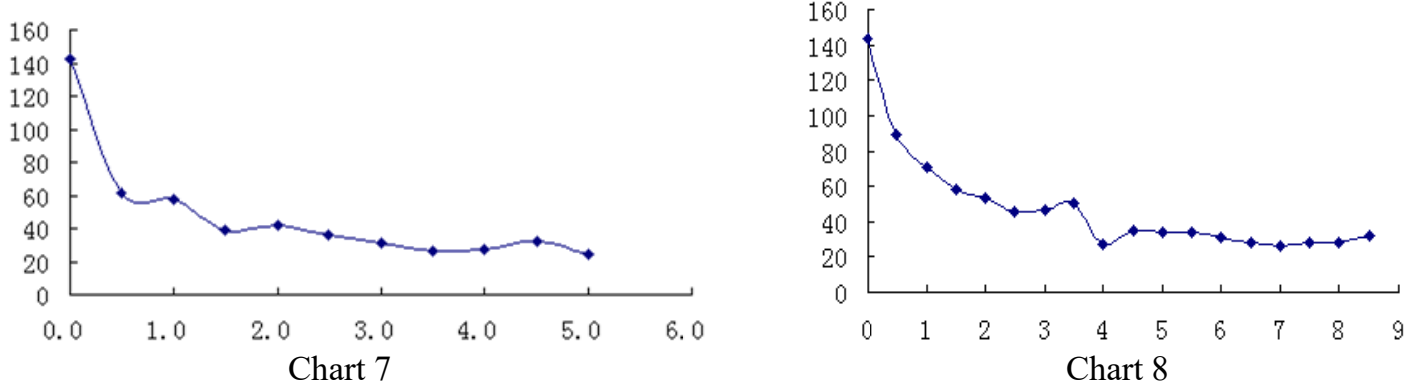

Deacidification of $3 \%$ titanium sulfate catalyzing $20 \%$ methanol

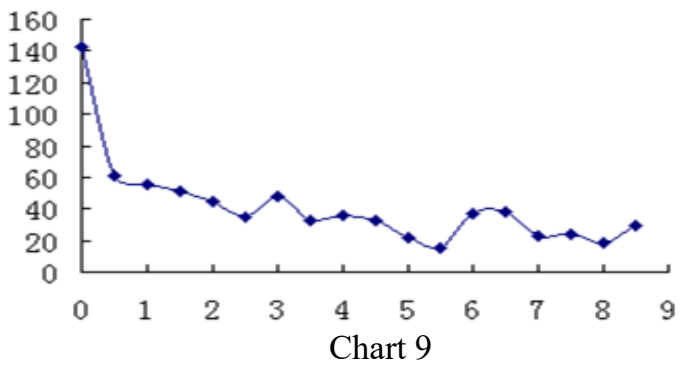

Fig.3 Trend line changes with time of 20\% amount of methanol and appropriate amount of glycerin deacidification acid value( $\mathrm{x}$ axis is time, $\mathrm{y}$ axis is acid value)

\section{Discussion of experimental results}

On the basis of the experimental data and chart, we analyze to get:

(1) Under the condition of $20 \%$ methanol dosage, three catalysts catalyzes glycerin and methanol to do esterification is slower than three catalysts directly catalyzes methanol.

(2)Acid value drops to 25 after sulfuric acid 20\% methanol esterification 5 hours' reaction, monohydrate sodium bisulfate after 5 hours' ,26, sulfuric acid after 5.5 hours', 16.(See Table 4,5 and Fig.4)

\section{Secondary esterification process optimization research of methanol and glycerin mixture}

The experimental data of $\mathbf{2 0 \%}$ amount of methanol andappropriate amount of glycerin deacidification

Table 4 The first step deacidification data table of methanol and glycerin mixture $(20 \%$ methanol $)$

\begin{tabular}{|c|l|r|r|r|r|r|r|r|}
\hline No. & $\begin{array}{c}\text { Time } \\
\text { catalyst }\end{array}$ & 0 & 0.5 & 1 & 1.5 & 2 & 2.5 & 3 \\
\hline 35 & Sulfuric acid & 143 & 105 & 93 & 88 & 87 & 78 & 77 \\
\hline 37 & Sodium bisulfate & 126 & 106 & 100 & 96 & 89 & 87 & 85 \\
\hline 39 & Titanium sulfate & 143 & 97 & 85 & 76 & 71 & 60 & 57 \\
\hline
\end{tabular}

Notice: time:hour; acid value unit is $\mathrm{mg} / \mathrm{g}$,according to $\mathrm{KOH}$; The reaction of methanol glycerin is secondary use of collecting waste liquid; Catalyst sodium bisulfate is monohydrate sodium bisulfate.

Table 5 The second step deacidification data table of methanol and glycerin mixture $(20 \%$ methanol $)$

\begin{tabular}{|c|l|c|c|c|c|c|c|c|c|c|}
\hline No. & Time & 0 & 0.5 & 1 & 1.5 & 2 & 2.5 & 3 & 3.5 & 4 \\
\hline 36 & Sulfuric acid & 87 & 57 & 18 & 14 & 9 & 9 & 11 & & \\
\hline 38 & Sodium bisulfate & 85 & 52 & 42 & 41 & 36 & 35 & 28 & 27 & 28 \\
\hline 40 & Titanium sulfate & 69 & 26 & 18 & 19 & 12 & 10 & 6 & & \\
\hline
\end{tabular}


Notice: time:hour; acid value unit is $\mathrm{mg} / \mathrm{g}$,according to $\mathrm{KOH}$; Catalyst sodium bisulfate is monohydrate sodium bisulfate.

Deacidification1 of $1.5 \%$ sulfuric acidcatalyzing $20 \%$ methanolDeacidification 2 of $1.5 \%$ sulfuric acidcatalyzing $20 \%$ methanol
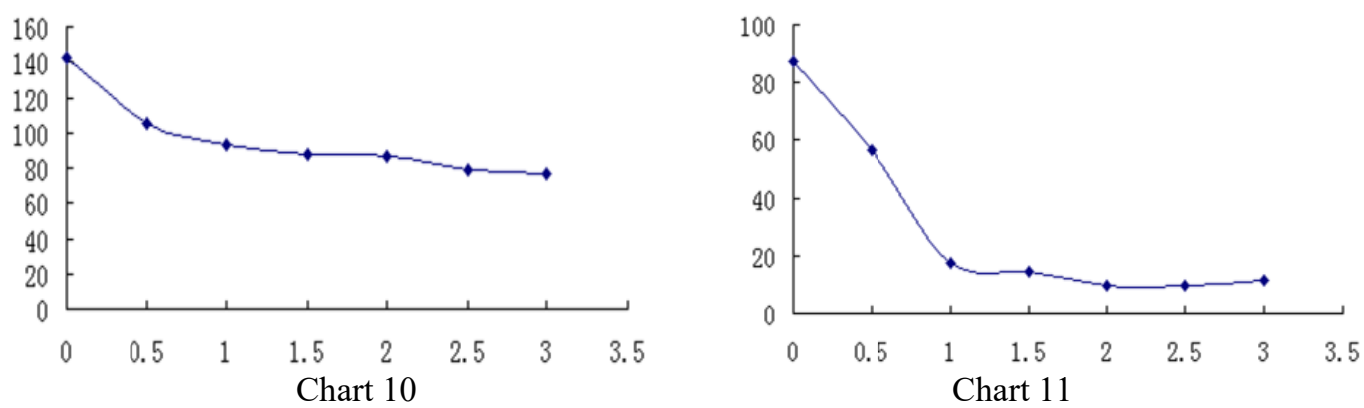

Deacidification1 of $4 \%$ sodium bisulfate catalyzing $20 \%$ methanolDeacidification 2 of $4 \%$ sodium bisulfate catalyzing $20 \%$ methanol
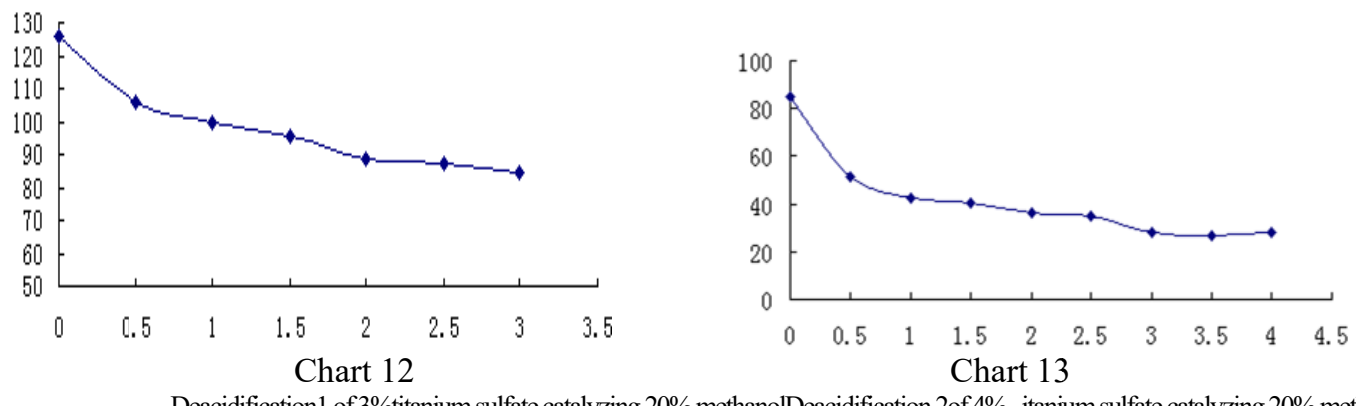

Deacidification 1 of $3 \%$ titanium sulfate catalyzing $20 \%$ methanolDeacidification 2of $4 \%$ itanium sulfate catalyzing $20 \%$ methanol
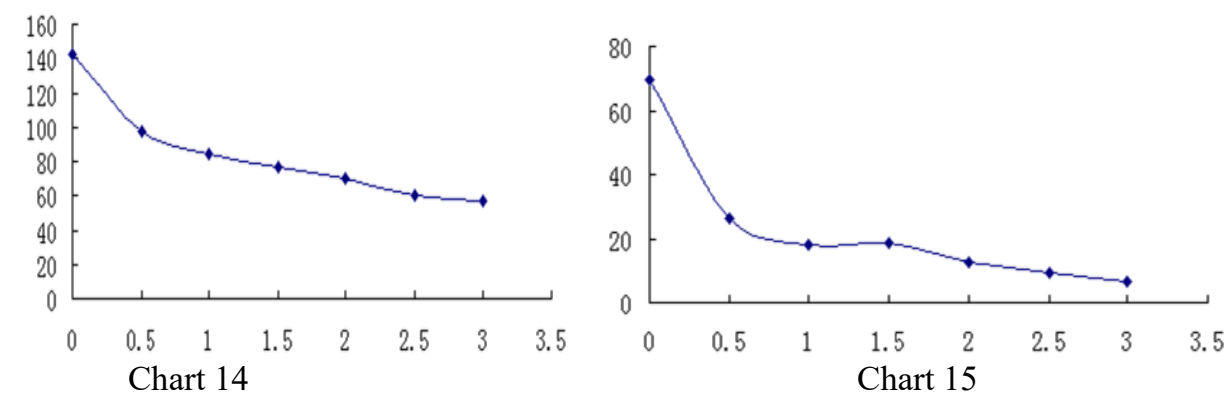

Fig. 4 Trend line changes with time of $20 \%$ amount of methanol and appropriate amount of glycerin deacidification acid value(x axis is time, $y$ axis is acid value)

\section{Discussion of experimental result}

According to the above data and chart analysis, under the shortest time and the best effects of deacidification principle, we've come to the conclusion that the dosage of $20 \%$ methanol condition, under the condition of sulfuric acid (1.5\%), titanium sulfate $(3 \%)$, monohydrate sodium hydrogen sulfate $(4 \%)$ of the three kinds of catalyst 's secondary esterification process:

The acid value of raw material oil is about $140 \mathrm{mg} / \mathrm{g}$

a.secondary esterification process under the condition of $1.5 \%$ sulfuric acid as catalyst catalyzing dosage of $20 \%$ methanol

Take oil according to the proportion of $20 \%$ methanol and add recycling methanol glycerin waste liquid, under the condition of fully mixing condenser heating 1.5 hours' reaction, acid value is about $88 \mathrm{mg} / \mathrm{g}$, then take upper oil liquid of separatory liquid, add the original oil $20 \%$ methanol and $1.5 \%$ sulfuric acid, under the condition of fully mixing condenser heating 1.5 hours' reaction, acid value dropped to about $14 \mathrm{mg} / \mathrm{g}$, add corresponding amount of glycerin separatory liquid with methanol, then recyle methanol glycerin waste liquid for reuse, so cycle.[7] 
b. secondary esterification process under the condition of $4 \%$ monohydrate sodium bisulfate as catalyst catalyzing dosage of $20 \%$ methanol

Take oil according to the proportion of $20 \%$ methanol and add recycling methanol glycerin waste liquid, under the condition of fully mixing condenser heating 2 hours' reaction, acid value is about $85 \mathrm{mg} / \mathrm{g}$, then take upper oil liquid of separatory liquid, add the original oil $20 \%$ methanol and $4 \%$ monohydrate sodium bisulfate, under the condition of fully mixing condenser heating 3 hours' reaction, acid value dropped to about $28 \mathrm{mg} / \mathrm{g}$, add corresponding amount of glycerin separatory liquid with methanol, then recyle methanol glycerin waste liquid for reuse, so cycle.

c.secondary esterification process under the condition of $3 \%$ Titanium sulfate as catalyst catalyzing dosage of $20 \%$ methanol

Take oil according to the proportion of $20 \%$ methanol and add recycling methanol glycerin waste liquid, under the condition of fully mixing condenser heating 2 hours' reaction, acid value is about $70 \mathrm{mg} / \mathrm{g}$, then take upper oil liquid of separatory liquid, add the original oil $20 \%$ methanol and 3\% Titanium sulfate, under the condition of fully mixing condenser heating 2 hours' reaction, acid value dropped to about $10 \mathrm{mg} / \mathrm{g}$, add corresponding amount of glycerin separatory liquid with methanol, then recyle methanol glycerin waste liquid for reuse, so cycle.

Above three process dropping acid results are better than with three kinds of catalyst respectively catalyzing the same amount of methanol esterification and methanol glyceride step alone , and time is shorter.[8]

\section{Conclusion and suggestion}

Through experiments, this paper analyzed and researched the deacidification situation of the different catalyst catalyzing methanol, glycerin alone and methanol glycerin mixture, and compared to research deacidification situation of inorganic and solid acid catalyst catalyzing methanol. In methanol esterification study, we found that effect of sulfuric acid catalytic acid reduction is good, then titanium sulfate. By single deacidification research of methanol and glycerin mixture, we found that under the same conditions, effect and progress of single deacidification of methanol and glycerin mixture is slower than single deacidification of methanol alone. Maybe due to large viscosity of glycerin and insufficient mixing, which makes mass transfer become limiting factor. [9] Through the experimental data, we found that reaction in an hour of methanol and glycerin mixture esterification and methanol esterification can both drop acid value below the original $1 / 2$ acid value, response speed is almost the same.

In methanol mixed with glycerin secondary esterification process optimization research, through the comparison of experimental data, we found that the mix methanol glycerin drop acid was divided into two stages, namely the first stage using methanol glycerin mixture (the recycling waste liquid of second step) dropping acid, the second step using methanol esterification drop acid alone. [10] This step used synthesis methanol esterification acid reduction method accelerate the dropping acid speed, change the dropping acid degree, and has realized the full use of raw materials. On the basis of the data, we summarized three acid reduction process of the sulfuric acid $(1.5 \%)$, titanium sulfate $(3 \%)$, monohydrate sodium hydrogen sulfate $(4 \%)$ of the three kinds of each catalyst of methanol $(20 \%)$ mixed with glycerin secondary esterification drop acid.

We suggest subsequent researchers to make further researches on the aspect of glycerine esterification alone, to seek a better catalyst, avoid or reduce the black phenomenon of reaction liquid, make the production convenient and feasible. Further explored and analyzed compound catalytic effect, seek environmental protection and efficient catalytic system, the optimize best reaction conditions of bettrer catalyst, and optimize dropping acid process. 


\section{References}

[1] Bin Liang. Production Technology of Biodiesel[J], Chemical Progress, 2005, 24(6): 577-585.

[2] Cheng-Ping Yang, Jian-Ming Yang, Jian Lv. The Synthesis and Using Research Progress of Biodiesel [J], Industrial Catalysis,2005,13(5):9-13.

[3] Hass M J. Improving the economics of biodiesel production hrough the use of low value lipids as feedstocks: Vegetablee oil soapstock [J],Fuel Processing Technology, 2005, 86(10): 1087-1096.

[4] Kalam MA, Masjuki HH.Biodiesel from palm oil-an analysis of itsproperties and potential[J],Biomass Bioenergy ,2002,23:471-9.

[5] Marchetti JM,Miguel VU, Errazu AF. Possible methods for biodiesel production[J], Renew Sust Energy Rev 2007,11:1300-11.

[6] ZhangY, DubeMA,McleanDD.Bidodiesel productiong from wastecooking oil:Process design and technologyical assessment[J],Biore source technology, 2003,89(1):1-16.

[7] Deng-Hua Yu.The research progress of biodiesel synthesis catalyst and process[J],Industrial catalysis,2006, 14(Supplement):76-79.

[8] Mao-Qing Huang, Ding-xian Lu. Production practice of high temperature gas phase method and supplement ester exchange process of producing fatty acid methyl ester[J],Daily-use chemical process,2000(1):44-47.

[9] TsinghuaUniversity.A two-step catalytic acid oil method of producing biodiesel:China,1840612A [P].2006-10-04.

[10] Jian-Zhong Yin,Min Xiao,Ji-Bin Song.Biodiesel from soybean oil in supercritical methanol with co-solvent [J],Energy Conversion and Management,49 (2008): 908-912. 